\title{
Pneumonia Organizativa Fibrinosa Aguda Induzida pelo Rituximab: Uma Entidade a (Re)conhecer
}

\section{Acute Fibrinous and Organizing Pneumonia Related to Rituximab: An Entity to Recognize}

Alexandra Couto ${ }^{1}$, Marta Almeida ${ }^{1}$, Herlander Marques ${ }^{1}$, Rui Nabiçơ ${ }^{1}$, Rui Rolo², João Cunha², Fernando Pardal ${ }^{3}$, Sofia Carvalho ${ }^{3}$

\section{RESUMO}

A pneumonia organizativa fibrinosa aguda é uma entidade histológica, incluída no grupo das doenças pulmonares intersticiais, com múltiplas etiologias, incluindo a toxicidade farmacológica.

Os autores descrevem o caso de um homem de 73 anos, com diagnóstico de linfoma linfoplasmocítico, sob tratamento de manutenção com rituximab após quimioterapia. O doente desenvolveu um quadro clínico insidioso caracterizado por tosse não produtiva, dispneia e astenia. A integração dos aspetos imagiológicos e da histologia de biópsia pulmonar conduziram ao diagnóstico de pneumonia organizativa fibrinosa aguda. Após suspensão do fármaco e instituição de corticoterapia o doente apresentou evolução clínica e imagiológica favorável.

Dada a utilização crescente de terapêuticas-alvo em oncologia, o presente caso pretende contribuir para o reconhecimento desta entidade como potencial evento adverso.

PALAVRAS-CHAVE: Pneumonia em Organização Criptogénica/diagnóstico; Pneumonia em Organização Criptogénica/induzida quimicamente; Pneumonias Intersticiais Idiopáticas; Rituximab/efeitos adversos

\section{ABSTRACT}

Acute fibrinous organizational pneumonia is a histological entity included in the group of interstitial lung diseases, with multiple etiologies including pharmacological toxicity.

The authors describe the case of a 73-year-old man diagnosed with lymphoplasmacytic lymphoma under maintenance treatment with rituximab after chemotherapy. The patient developed an insidious clinical picture characterized by non-productive cough, dyspnea and asthenia. The integration of imaging aspects and pulmonary biopsy histology led to the diagnosis of acute fibrinous and organizing pneumonia. After suspension of the drug and institution of corticosteroids, the patient presented a favorable clinical and imaging course. 
Given the increasing use of targeted therapies in oncology, the present case aims to contribute to the recognition of this entity as a potential adverse event.

KEYWORDS: Cryptogenic Organizing Pneumonia/chemically induced; Cryptogenic Organizing Pneumonia/diagnosis; Idiopathic Interstitial Pneumonias; Rituximab/adverse effects

\section{INTRODUÇÃO}

A pneumonia organizativa fibrinosa aguda (acute fibrinous and organizing pneumonia - AFOP) é uma doença pulmonar rara, descrita pela primeira vez por Beasley et al ${ }^{1} \mathrm{em} 2002$, caracterizada pela presença difusa de fibrina intra-alveolar com pneumonia organizativa associada. Em 2013, num documento conjunto elaborado pela Sociedade Torácica Americana (ATS) e Sociedade Respiratória Europeia (ERS), a AFOP foi considerada um padrão histológico raro de pneumonia intersticial. ${ }^{2} \mathrm{As} \mathrm{ca-}$ racterísticas histológicas particulares que a tornam uma entidade distinta são a presença de depósitos de fibrina nos alvéolos pulmonares com múltiplos focos de atividade fibroblástica, mas ausência das habituais membranas hialinas e de eosinófilos. Os achados imagiológicos são idênticos ao da pneumonia organizativa criptogénica, com opacidades alveolares difusas habitualmente com uma distribuição periférica e multilobar.

A AFOP pode ser idiopática ou estar associada a doenças autoimunes e do tecido conjuntivo, agentes infeciosos e reações adversas a fármacos. ${ }^{3} \mathrm{O}$ diagnóstico precoce é importante, pois é reconhecido que esta entidade pode ter uma evolução progressiva e fatal. Existem duas formas da doença, uma mais grave que cursa rapidamente com insuficiência respiratória e uma forma subaguda com melhor prognóstico. ${ }^{4}$ Os sintomas pulmonares mais comuns incluem tosse, dispneia e febre. Focos de consolidação e opacidades em vidro-despolido são os achados imagiológicos mais precoces. ${ }^{5}$

Com a emergência das terapêuticas-alvo e da imunoterapia, tem aumentado o número de casos descritos de doença pulmonar intersticial decorrentes da terapêutica antineoplásica, sendo rara a referência à AFOP. ${ }^{\circ}$ O rituximab é um anticorpo monoclonal que tem como alvo a proteína CD20, expressa à superfície dos linfócitos B, com indicação terapêutica em muitos tipos de linfomas não- Hodgkin (LNH). Apesar de ser uma complicação muito rara, têm sido descritos vários padrões de lesão pulmonar aguda associada ao rituximab, com apenas um caso relatado com o padrão histológico característico da AFOP?

\section{CASO CLÍNICO}

Os autores descrevem o caso de um doente do sexo masculino, de 73 anos, caucasiano, autónomo nas ativi- dades da vida diária. Apresentava antecedentes patológicos de hipertensão arterial, dislipidemia e hepatite $\mathrm{B}$ crónica. Ex-fumador de 20 unidades-maço-ano (cessação tabágica há 35 anos). Encontrava-se medicado em ambulatório com atorvastatina $20 \mathrm{mg} / \mathrm{dia}$, pantoprazol $40 \mathrm{mg} / \mathrm{dia}$, piribedil $50 \mathrm{mg} / \mathrm{dia}$, valsartan/hidroclorotiazida 160/12,5 mg/dia e tenofovir $300 \mathrm{mg} / \mathrm{dia}$.

Efetuado o diagnóstico de LNH linfoplasmocítico em janeiro de 2015, tendo iniciado quimioterapia com esquema R-CVP (rituximab-ciclofosfamida-vincristina-prednisolona). Realizou seis ciclos até junho de 2015, mantendo posteriormente terapêutica de manutenção com rituximab.

Em agosto de 2016, após cinco ciclos de rituximab em monoterapia, apresentou quadro clínico caracterizado por tosse não produtiva, dispneia grau 2, de acordo com a escala Medical Research Council (mMRC) e astenia; ocasionalmente apresentava-se sub-febril. Referia evolução insidiosa dos sintomas com agravamento progressivo desde há um ano. Ao exame objetivo estava eupneico em ar ambiente, sem sinais de dificuldade respiratória e com saturação periférica de oxigénio de 96\%; a auscultação pulmonar evidenciava sibilância expiratória dispersa e crepitações bibasais.

Analiticamente não apresentava aumento dos parâmetros inflamatórios, as serologias para o vírus da imunodeficiência humana (VIH) e citomegalovírus (CMV) eram negativas e foram também negativos os marcadores de autoimunidade.

A tomografia computorizada (TC) do tórax evidenciou múltiplos nódulos pulmonares e focos de densificação em vidro despolido e zonas de consolidação com tendência à organização, bilaterais e com predomínio nos lobos inferiores (Fig. 1).

Foram admitidas várias hipóteses diagnósticas, nomeadamente pneumonia infeciosa, pneumonite de hipersensibilidade, doença pulmonar intersticial secundária a fármacos, pneumonia organizativa criptogénica (COP), insuficiência cardíaca ou tromboembolismo pulmonar (TEP).

Foi realizada broncoscopia flexível, que não revelou alterações. Os exames microbiológicos realizados (antigenúria para Streptococcus pneumoniae e Legionella pneumophila, hemoculturas e lavado broncoalveolar) não identi- 

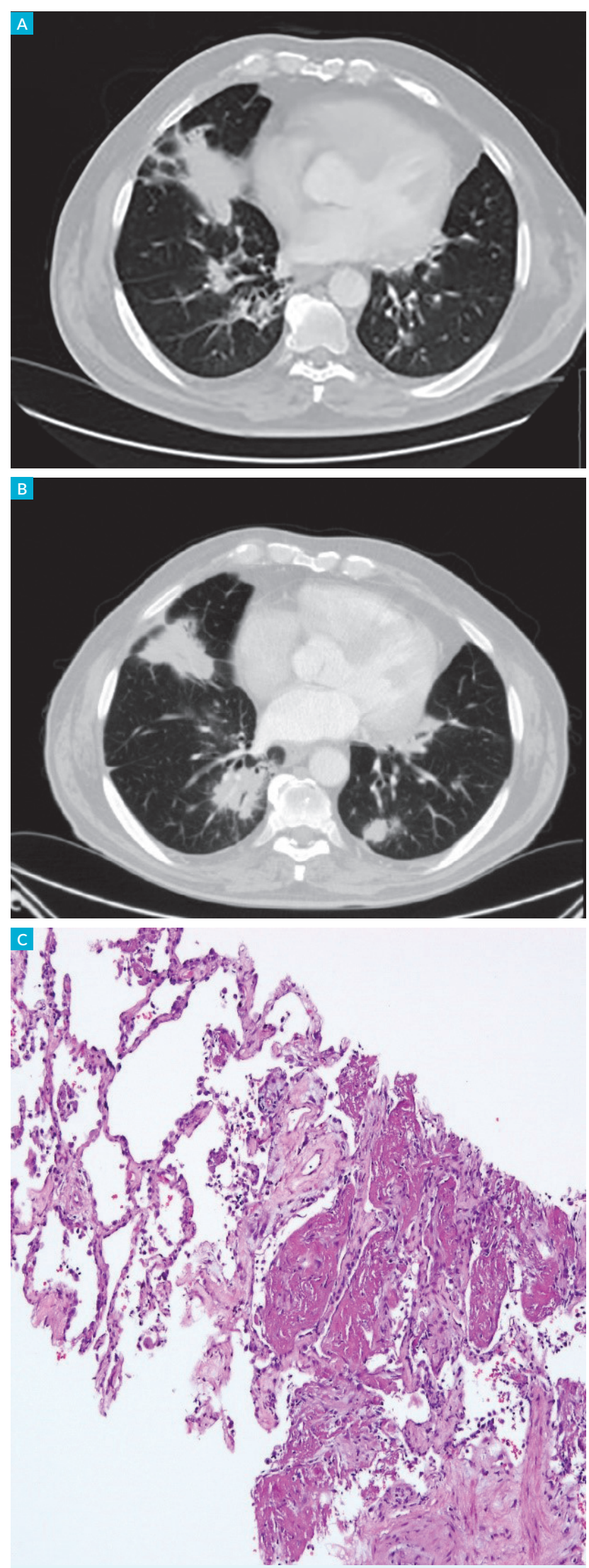

FIGURA 1. Pneumonia organizativa fibrinosa aguda. TC do tórax evidenciando focos de densificação em vidro despolido e zonas de consolidação, bilaterais e com predomínio basal (A) e aspeto imagiológico após três meses de terapêutica com corticoide, onde se verifica diminuição das zonas de densificação do parênquima pulmonar (B). Aspeto histológico da biópsia pulmonar (C) onde se observa proliferação de células miofibroblásticas e ocupação parcial de alguns espaços alveolares por tecido de granulação e outros preenchidos por nódulos de fibrina (H\&E, 100x). ficaram nenhum agente infecioso. Foi também excluída infeção por Mycobacterium tuberculosis e Pneumocystis jirovecii. $\bigcirc$ ecocardiograma transtorácico não demonstrou alterações da função cardíaca.

Foi suspensa a terapêutica com rituximab por suspeita de toxicidade pulmonar associada ao fármaco. $\bigcirc$ doente foi referenciado para a consulta de Pneumologia, tendo realizado biópsia transtorácica. $\bigcirc$ exame histológico revelou áreas de fibrose e proliferação de células miofibroblásticas, com ocupação parcial de espaços alveolares por tecido de granulação e alvéolos preenchidos por "nódulos" de fibrina (Fig. 1); aspetos morfológicos compatíveis com pneumonia organizativa fibrinosa aguda.

O estudo funcional respiratório demonstrou uma síndrome ventilatória obstrutiva das pequenas vias aéreas, com diminuição ligeira da capacidade de transferência alvéolo-capilar do CO por single breath, normal quando corrigida pelo volume alveolar.

O doente iniciou corticoterapia com prednisolona na dose de 0,5 mg/kg/dia, correspondente a $40 \mathrm{mg} / \mathrm{dia}$, com progressiva melhoria clínica. Os achados imagiológicos três meses após início de corticoide mostraram diminuição das dimensões dos nódulos bem como das áreas de densificação do parênquima pulmonar (Fig. 1).

Atualmente, o doente mantém seguimento em consulta de Oncologia e Pneumologia, com reavaliação clínica e imagiológica, mantendo necessidade de corticoterapia oral com prednisolona 15 mg/dia, em esquema de desmame lento.

\section{DISCUSSÃO}

A fisiopatologia da doença pulmonar intersticial induzida pela terapêutica antineoplásica não é ainda totalmente compreendida. Adicionalmente à toxicidade do fármaco, fatores genéticos e intrínsecos do hospedeiro poderão estar implicados, uma vez que apenas uma minoria dos doentes expostos desenvolve lesão pulmonar sintomática. As terapêuticas-alvo, como os anticorpos monoclonais, podem virtualmente induzir todos os padrões histopatológicos de pneumonia intersticial, incluindo pneumonite de hipersensibilidade, pneumonia organizativa, pneumonia eosinofílica, pneumonite granulomatosa, hemorragia alveolar ou dano alveolar difuso. ${ }^{8}$

Tendo em conta as potenciais etiologias e a apresentação clínica heterogénea, a incidência da AFOP está provavelmente subestimada. No caso particular dos fármacos antineoplásicos, existem lacunas no conhecimento dos mecanismos fisiopatológicos subjacentes ao desenvolvimento da AFOP, o que tem dificultado o seu 
reconhecimento na prática clínica diária. Esta entidade tem sido descrita como uma reação não específica do parênquima pulmonar, com uma evolução clínica que é influenciada pela gravidade da neoplasia subjacente. ${ }^{9}$ As neoplasias que conferem maior risco para o desenvolvimento da AFOP são as hematológicas, nomeadamente os linfomas, as síndromes mielodisplásicas, a leucemia mieloide aguda e a leucemia linfoblástica aguda. ${ }^{10}$

São escassos os relatos de casos de AFOP, pelo que permanece ainda alguma ambiguidade quanto à sua caracterização clínica, imagiológica e aspetos terapêuticos e de prognóstico. $O$ diagnóstico é um desafio e assenta na integração dos dados clínicos, radiológicos e anatomopatológicos. As manifestações clínicas e imagiológicas inespecíficas evocam outros diagnósticos, nomeadamente a pneumonia infeciosa, que poderão conduzir à instituição desnecessária de antibioterapia. Acresce também o facto de poder existir uma dissociação temporal entre o início do fármaco e as manifestações clínicas. Não existe tratamento específico e usualmente a terapêutica com corticosteroides é instituída de forma empírica, com boa resposta clínica.

Os autores consideram ser útil a descrição do caso clínico e o destaque das suas particularidades para que a AFOP seja uma entidade a considerar nos diagnósticos diferenciais de lesão pulmonar. No contexto específico da Oncologia, em que novas terapêuticas conduzirão inevitavelmente a novos padrões de efeitos adversos, o relato de casos irá proporcionar um melhor conhecimento da doença pulmonar intersticial secundária à terapêutica antineoplásica.

CONFLITOS DE INTERESSE: Os autores declaram não ter qualquer conflito de interesse na realização do presente trabalho.

FONTES DE FINANCIAMENTO: Não houve qualquer fonte de financiamento na realização do presente trabalho.

CONFIDENCIALIDADE DOS DADOS: Os autores declaram ter seguido os protocolos da sua instituição acerca da publicação dos dados de doentes.

PROTEÇÃO DE PESSOAS E ANIMAIS: Os autores declaram que os procedimentos seguidos na elaboração do presente trabalho estão em conformidade com as normas das comissões de investigação clínica e de ética, bem como da declaração de Helsínquia e da Associação Médica Mundial.
CONFLICTS OF INTEREST: The authors declare that they have no conflicts of interest.

FINANCIAL SUPPORT: This work has not received any contribution, grant or scholarship.

CONFIDENTIALITY OF DATA: The authors declare that they have followed the protocols of their work center on the publication of data from patients.

PROTECTION OF HUMAN AND ANIMAL SUBJECTS: The authors declare that the procedures followed were in accordance with the regulations of the relevant clinical research ethics committee and with those of the Code of Ethics of the World Medical Association (Declaration of Helsinki).

\section{REFERÊNCIAS}

1. Beasley MB, Franks TJ, Galvin JR, Gochuico B, Travis WD. Acute fibrinous and organizing pneumonia: A histological pattern of lung injury and possible variant of diffuse alveolar damage. Arch Pathol Lab Med. 2002;126:1064-70.

2. Travis WD, Costabel U, Hansell DM, King TE Jr, Lynch DA, Nicholson AG, et al. An official American thoracic society/European respiratory society statement: Update of the international multidisciplinary classification of the idiopathic interstitial pneumonias. Am J Respir Crit Care Med. 2013;188:733-48.

3. Saxena P, Kumar K, Mittal S, Goyal N, Trikha S, Vashisth A. Acute fibrinous and organizing pneumonia: A rare form of nonbacterial pneumonia. Indian J Crit Care Med. 2016;20:245-7.

4. Xu XY, Chen F, Chen C, Sun HM, Zhao BL. Acute fibrinous and organizing pneumonia: A case report and literature review. Exp Ther Med. 2016;12:3958-62.

5. Dai JH, Li H, Shen W, Miao LY, Xiao YL, Huang M, et al. Clinical and radiological profile of acute fibrinous and organizing pneumonia: a retrospective study. Chin Med J. 2015;128:2701-6.

6. Gupta A, Sen S, Naina H. Acute fibrinous and organising pneumonia: a rare histopathological variant of chemotherapy-induced lung injury. BMJ Case Rep. 2016;2016.

7. Victor N. Acute fibrinous organizing pneumonia: rituximab induced fibrin balls within alveoli, an unknown entity. Am J Respir Crit Care Med. 2016;194:A1596.

8. Kokosi MA, Nicholson AG, Hansell DM, Wells AU. Rare idiopathic interstitial pneumonias: LIP and PPFE and rare histologic patterns of interstitial pneumonias: AFOP and BPIP. Respirology. 2016;21:600-14.

9. Gomes R, Padrão E, Dabó H, Soares Pires F, Mota P, Melo $N$, et al.. Acute fibrinous and organizing pneumonia: A report of 13 cases in a tertiary university hospital. Medicine. 2016;95:e4073.

10. Kuza C, Matheos T, Kathman D, Heard SO. Life after acute fibrinous and organizing pneumonia: a case report of a patient 30 months after diagnosis and review of the literature. J Crit Care. 2016;31:255-61. 